\title{
Edge-illumination X-ray dark-field imaging for visualising defects in composite structures
}

\author{
M. Endrizzia ${ }^{\mathrm{a}}$, B.I.S. Murat ${ }^{\mathrm{b}}$, P. Fromme ${ }^{\mathrm{b}}$, A. Olivo ${ }^{\mathrm{a}}$ \\ ${ }^{a}$ Department of Medical Physics and Biomedical Engineering, University College London, \\ Gower Street, WC1E 6BT London, United Kingdom \\ ${ }^{b}$ Department of Mechanical Engineering, University College London, WC1E 7JE, \\ London, UK
}

\begin{abstract}
We report on a novel X-ray imaging technique enabling the detailed visualization of the consequences of impact damage in composite materials. The system is based on edge-illumination and can be implemented with conventional X-ray equipment, namely a rotating anode source and a digital detector, with the addition of two achromatic optical elements. It is robust against vibrations and thermal stress and works well with a polychromatic energy spectrum. The system is multi-modal and provides three representations of the sample: absorption, differential phase and dark-field. The latter is of particular interest to detect cracks and voids of dimensions that are smaller than actual spatial resolution of the imaging system. An example of application to a carbon fibre laminate plate consisting of 8 layers with a symmetric layup sequence of $[0 / 90]$ is presented. Low-velocity impact damage was induced using standard drop weight procedures. The plates and impact damage were initially characterized using ultrasonic measurements and the two imaging methods are compared one to each other. Depending on the severity of the impact, fiber and matrix breakage and delamination occurred.
\end{abstract}

Keywords: X-ray phase-contrast imaging, Dark-field imaging, Composite structures, Impact damage

\section{Introduction}

X-ray Phase-Contrast Imaging (XPCI) enables to overcome the limitations of conventional radiography related to the lack of contrast for weakly absorbing details. This is achieved by introducing sensitivity also to the 
phase shifts imparted to the X-ray beam by the sample [1]. XPCI finds application in a wide range of fields, encompassing biological and medical sciences, security inspection and materials science. Several approaches exist for obtaining phase-contrast images in the X-ray regime, using large-scale synchrotron facilities and more compact X-ray-tube-based equipment [2-12]. Details on the imaging methods, developments and their applications are covered in recent reviews on the subject [13, 14]. Edge-illumination (EI) [9] and its area imaging implementation, often called coded-aperture [15], are XPCI techniques providing quantitative amplitude and phase retrieval [16]. EI was adapted to use several types of radiation sources, namely synchrotron radiation [9], rotating anode [15, 16] and microfocal [17] X-ray tubes. Ultrasmall-angle X-ray scattering imaging, in the hard X-rays regime was also recently developed for an EI set-up [18], resulting in the extraction of a third representation of the sample, also referred to as dark-field image.

We present here the application of EI dark-field imaging to the visualisation of the defects occurring in a composite plate after impact damage. We start describing the sample, the X-ray dark-field imaging system and the imaging method. We present the images obtained by means of such a system, also compared to what can be achieved using conventional radiography. In addition, a comparison with ultrasound imaging is also presented and their complementarity is discussed.

\section{Materials and methods}

The typical experimental set-up for an EI imaging system is composed of an X-ray source, a sample masks that shapes the beam before it interacts with the sample, and an analyser which is composed of a second mask and a digital detector. A sketch of the system is shown in Fig. 1. The parameters used for this experiment are as follows. The source to detector distance was $z_{s d}=2 \mathrm{~m}$ while the sample to detector distance was $z_{o d}=40 \mathrm{~cm}$, with a geometrical magnification of 1.25. The source was a molybdenum target, rotating anode X-ray tube (Rigaku, MM007), operated at $40 \mathrm{kVp}$ and $20 \mathrm{~mA}$, with a source size of approximately $70 \mu \mathrm{m}$. The pitches of the two masks are $p_{1}=67$ and $p_{2}=83.5 \mu \mathrm{m}$, and the apertures $a_{1}=12$ and $a_{2}=20 \mu \mathrm{m}$, for the sample and detector mask respectively. The masks were manufactured to the authors' design by Creatv Microtech (Potomac, MD) and had a gold thickness of about $30 \mu \mathrm{m}$ and offered a field of view of about $5 \times 5 \mathrm{~cm}$. The alignment of the system [19] was performed by means of two stacks 


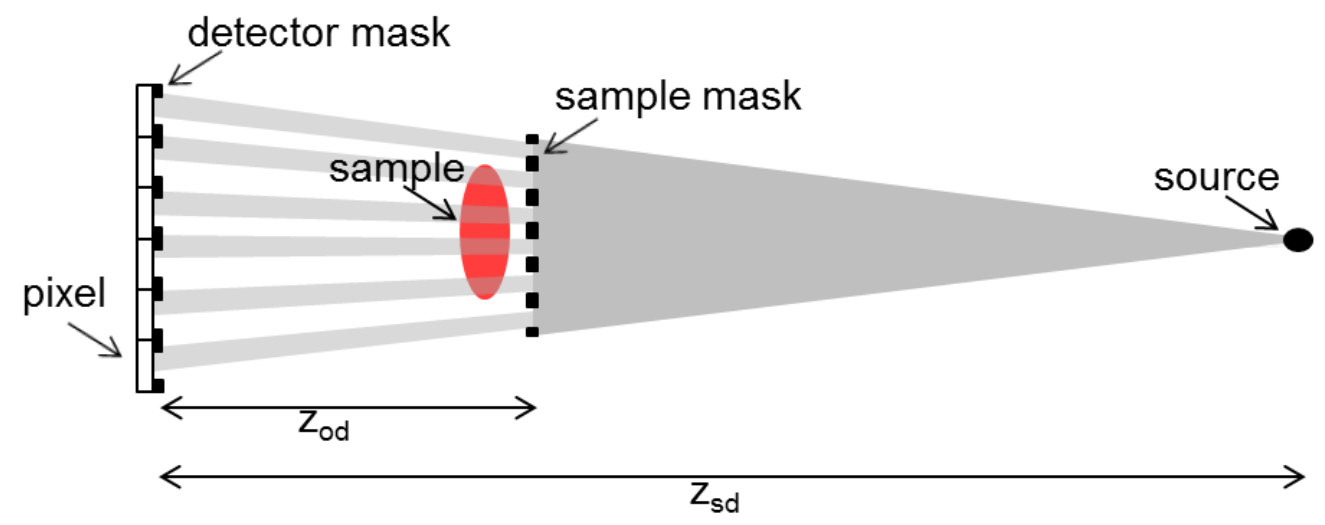

Figure 1: Sketch of the EI imaging system used for this experiment. A rotating anode Xray tube produces a polychromatic beam that is shaped into an array of laminar beamlets before the sample. After interaction with the sample, each beamlet is analised by means of the edge of a second mask, placed right in front of the detector.

of Newport (ILS150, MFA and SR50) and Kohzu (SA07A-RM) motorized stages.

A description of image formation based on geometrical optics can be used for a typical laboratory set-up [18]:

$$
\frac{I(x)}{I_{0}}=(L * O)\left(x-\Delta x_{R}\right) t
$$

where $I_{0}$ represent the intensity passing the sample aperture, $L$ is the illumination function characterising the system, $*$ denotes convolution and $O$ is the scattering distribution induced by the sample. $\Delta x_{R}=-\Delta \theta_{R} z_{\text {od }}$ is the beam shift caused by refraction in the sample $\Delta \theta_{R}$ and $t$ is the fraction of transmitted intensity. In order to retrieve the sample properties from the convolution Eq. 1, the following analytical solutions can be used [18]

$$
\left\{\begin{aligned}
t & =\frac{2 x_{1}}{A_{M N}} \sqrt{\frac{\pi}{D+C}} I_{2} \exp \left[\frac{1}{2^{4}} \frac{(D-C)^{2}}{D+C}\right] \\
\Delta x_{R} & =\frac{x_{1}}{2} \frac{D-C}{D+C} \\
\sigma_{M}^{2} & =\frac{2 x_{1}^{2}}{D+C}-\sigma_{N}^{2}
\end{aligned}\right.
$$

where $C=-2 \ln \left(I_{1} / I_{2}\right)$ and $D=-2 \ln \left(I_{3} / I_{2}\right)$, and a single Gaussian term is assumed for both $L(x)$ and for $O(x)$. $I_{1}$ and $I_{2}$ are acquired by using a lateral shift of $x_{1}= \pm 12 \mu \mathrm{m}$ of the sample mask while $I_{2}$ is acquired at $x_{1}=0$, 


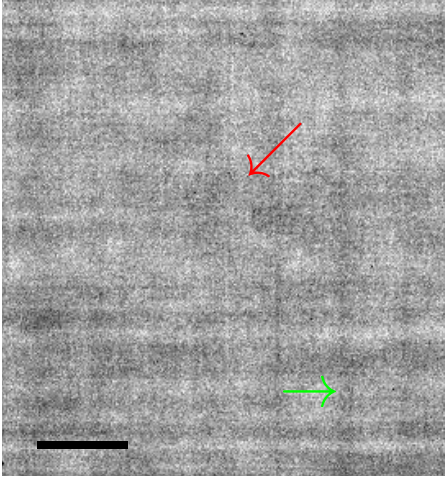

(a)

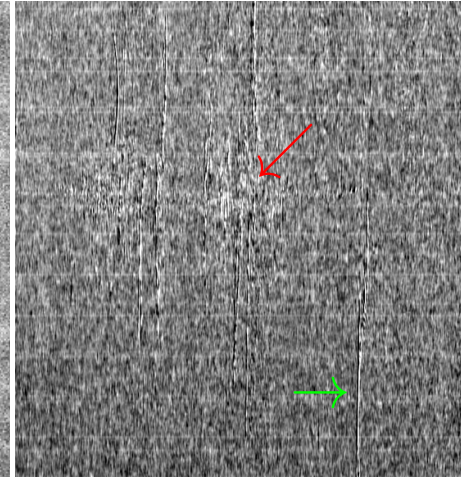

(b)

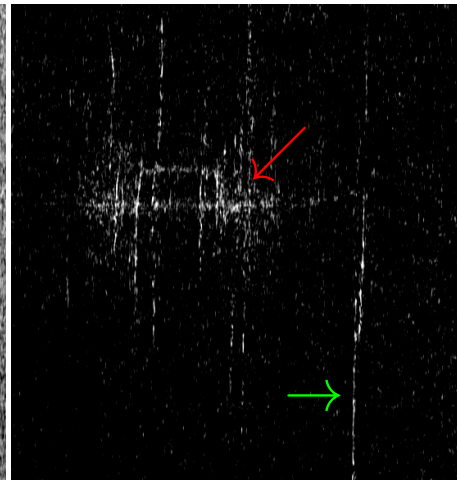

(c)

Figure 2: X-ray images of the damaged area in the composite plate. (a) Conventional radiography, (b) differential phase and (c) dark-field image. The scale bar is $1 \mathrm{~cm}$. The defects occurring in the plate after the impact damage are well detectable in the dark-field and differential phase images, while no apparent signal can be identified in the conventional radiography one.

i.e. in the position were the sample mask and the detector mask have aligned apertures and therefore the maximum intensity is transmitted. The parameters describing the sample $t, \Delta x_{R}$ and $\sigma_{M}^{2}$ can also be iteratively refined [20], and corrected for the inaccuracies arising from the masks' imperfections.

\section{Results}

The images obtained by means of EI X-ray phase contrast imaging are compared to what is observed with conventional radiography in Fig. 2. Defects in the inner structure of the plate, resulting from the impact, can be clearly visualized in the dak-field, Fig. 2(c). The larger cracks are well visible also in the differential phase image, Fig. 2(b), but only those one that follow the direction of the aperture. It is in fact a peculiar property of this imaging technique that the signal of an edge-like feature appears modulated, in the differential phase image, by the relative angle between the edge and the aperture and the edge; it is maximum when they are aligned and minimum when they are orthogonal to each other. A strong signal is observed both in the differential phase and in the dark-field images few centimetres away from the impact point. This is probably generated by 


\section{Conclusion}

\section{References}

[1] R. Fitzgerald, Phase-sensitive x-ray imaging, Physics Today 53 (7) (2000) 23-26.

[2] U. Bonse, M. Hart, An x-ray interferometer, Appl. Phys. Lett. 6 (1965) $155-156$.

[3] K. Goetz, E. Foerster, P. Zaumseil, M. P. Kalashnikov, I. A. Mikhailov, G. V. Sklizkov, S. I. Fedotov, Measurements of the parameters of shell targets for laser thermonuclear fusion using an X-ray schlieren method, Kvantovaia Elektronika Moscow 6 (1979) 1037-1042.

[4] A. Snigirev, I. Snigireva, V. Kohn, S. Kuznetsov, I. Schelokov, On the possibilities of x-ray phase contrast microimaging by coherent highenergy synchrotron radiation, Rev. Sci. Instrum. 66 (12) (1995) 54865492 .

[5] T. J. Davis, D. Gao, T. E. Gureyev, A. W. Stevenson, S. W. Wilkins, Phase-contrast imaging of weakly absorbing materials using hard X-rays, Nature 373 (1995) 595-598. doi:10.1038/373595a0.

[6] V. N. Ingal, E. A. Beliaevskaya, X-ray plane-wave topography observation of the phase contrast from a non-crystalline object, J. Phys. D Appl. Phys. 28 (1995) 2314-2317. doi:10.1088/0022-3727/28/11/012.

[7] S. W. Wilkins, T. E. Gureyev, D. Gao, A. Pogany, A. W. Stevenson, Phase-contrast imaging using polychromatic hard x-rays, Nature 384 (1996) 335-338.

[8] D. Chapman, W. Thomlinson, R. E. Johnston, D. Washburn, E. Pisano, N. Gmür, Z. Zhong, R. Menk, F. Arfelli, D. Sayers, Diffraction enhanced x-ray imaging, Phys. Med. Biol. 42 (1997) 2015-2025. doi:10.1088/00319155/42/11/001.

[9] A. Olivo, F. Arfelli, G. Cantatore, R. Longo, R. H. Menk, S. Pani, M. Prest, P. Poropat, L. Rigon, G. Tromba, E. Vallazza, E. Castelli, An innovative digital imaging set-up allowing a low-dose approach to phase contrast applications in the medical field, Med. Phys. 28 (2001) 1610-1619. doi:10.1118/1.1388219. 
[10] C. David, B. Nohammer, H. H. Solak, E. Ziegler, Differential x-ray phase contrast imaging using a shearing interferometer, Applied Physics Letters 81 (17) (2002) 3287-3289. doi:10.1063/1.1516611.

URL http://link.aip.org/link/?APL/81/3287/1

[11] L. Rigon, H.-J. Besch, F. Arfelli, R.-H. Menk, G. Heitner, H. PlothowBesch, A new DEI algorithm capable of investigating sub-pixel structures, J. Phys. D Appl. Phys. 36 (26) (2003) A260000-A112. doi:10.1088/0022-3727/36/10A/322.

[12] F. Pfeiffer, T. Weitkamp, O. Bunk, C. David, Phase retrieval and differential phase-contrast imaging with low-brilliance X-ray sources, Nat. Phys. 2 (2006) 258-261. doi:10.1038/nphys265.

[13] S. Wilkins, Y. Nesterets, T. Gureyev, S. Mayo, A. Pogany, A. Stevenson, On the evolution and relative merits of hard x-ray phase-contrast imaging methods, Phil. Trans. R. Soc. A 372: 20130021.

[14] A. Bravin, P. Coan, P. Suortti, X-ray phase-contrast imaging: from preclinical applications towards clinics, Physics in Medicine and Biology 58 (1) (2013) R1.

URL http://stacks.iop.org/0031-9155/58/i=1/a=R1

[15] A. Olivo, R. Speller, A coded-aperture technique allowing x-ray phase contrast imaging with conventional sources, Appl. Phys. Lett. 91 (7) (2007) 074106.

[16] P. R. Munro, K. Ignatyev, R. D. Speller, A. Olivo, Phase and absorption retrieval using incoherent X-ray sources, Proc. Natl. Acad. Sci. USA 109 (35) (2012) 13922-13927.

[17] M. Endrizzi, F. A. Vittoria, P. C. Diemoz, R. Lorenzo, R. D. Speller, U. H. Wagner, C. Rau, I. K. Robinson, A. Olivo, Phase-contrast microscopy at high x-ray energy with a laboratory setup, Optics Letters 39 (11) (2014) 3332-3335.

[18] M. Endrizzi, P. C. Diemoz, T. P. Millard, J. L. Jones, R. D. Speller, I. K. Robinson, A. Olivo, Hard x-ray dark-field imaging with incoherent sample illumination, Applied Physics Letters 104 (2) (2014) 024106. 
[19] T. P. Millard, M. Endrizzi, K. Ignatyev, C. K. Hagen, P. R. T. Munro, R. D. Speller, A. Olivo, Method for automatization of the alignment of a laboratory based x-ray phase contrast edge illumination system, Review of Scientific Instruments 84 (8) (2013) -.

[20] M. Endrizzi, A. Olivo, Absorption, refraction and scattering retrieval with an edge-illumination-based imaging setup, Journal of Physics D: Applied Physics 47 (50) (2014) 505102. 